\title{
Sensibilidad del TTPa en la detección de una deficiencia de factores de coagulación
}

\author{
aPTT sensitivity in the detection of coagulation factors deficiency
}

\author{
Milton Lombana1', Gloria Ramos', Ana Milena Torres², Pablo Sanabria³
}

'Servicio de Hematología y Oncología, Hospital Militar Central, Universidad Militar Nueva Granada (Bogotá, Colombia).

Departamento de Epidemiologia, Universidad El Bosque (Bogotá, Colombia).

3Departamento de Bioestadistica, Facultad de Medicina, Universidad Militar Nueva Granada (Bogotá, Colombia).

\section{Resumen}

Objetivos: Determinar la sensibilidad del TTPa con el reactivo Actin FSL para la detección de pacientes con déficit de factores de coagulación comparada con el estándar de referencia, que es la determinación directa del nivel de actividad de factores de coagulación por método coagulométrico de un paso.

Métodos: Población: pacientes del Hospital Militar Central (Bogotá, Colombia) con determinación de TTPa por Actin FSL y de niveles de factores de coagulación solicitados por sospecha de trastornos de hemostasia durante el período de junio del 2009 a febrero del 2011. Tipo de estudio: analítico de corte transversal de validación de pruebas diagnósticas. A todos los pacientes se les aplicó la prueba por evaluar, TTPa con Actin FSL y el estándar de referencia, de manera independiente a sus resultados.

Resultados: De junio del 2009 a febrero del 2011, se incluyeron 133 pacientes con realización de TTPa por Actin FSL y medición de factores de coagulación de manera concomitante. En la población total estudiada, la sensibilidad del TTPa fue del $98.7 \%$ con un LR- de 0.02 . Ningún paciente con deficiencia severa de factores de coagulación (definida como un valor $<1 \%$ ) presentó un valor de TTPa $<32.1 \mathrm{seg}$. El análisis por regresión lineal demostró que no existe relación entre el porcentaje de actividad de los factores de coagulación y el valor del TTPa en segundos.

Conclusiones: El TTPa con Actin FSL bajo una técnica estandarizada tiene alta sensibilidad en la detección de disminución de factores de coagulación, y un resultado normal hace muy improbable encontrar déficit de algún factor, por determinación directa.

Palabras clave: Diagnóstico, tiempo de tromboplastina parcial, factores de coagulación sanguínea.

\begin{abstract}
Objectives: Determine the sensitivity of aPTT reagent with Actin FSL for the detection of patients with clotting factor deficiency compared with the reference standard which is the direct determination of the level of coagulation factor activity by a coagulometric method at one step.

Methods: Population: Patients from the Hospital Militar Central (Bogotá, Colombia) with aPTT determination by Actin FSL and clotting factor levels requested for suspected disorders of hemostasis during the period June 2009 to February 2011. Type of study: Analytic cross sectional of validation of diagnostic tests.

All patients were administered the test to evaluate for Actin FSL aPTT and the gold standard, independently of their results. Results: From June 2009 to February 2011 were included 133 patients with aPTT by conducting Actin FSL and measurement of clotting factors concurrently. In the total study population, the sensitivity of the aPTT was $98.7 \%$ with a negative LR of 0.02 . No patient with severe deficiency of coagulation factors (defined as a value $<1 \%$ ) had a value of aPTT $<32.1$ seg. Linear regression analysis showed no correlation between the percentage of activity of coagulation factors and the aPTT value in seconds.

Conclusions: The sensitivity of aPTT with Actin FSL under a standardized technique has high sensitivity in the detection of decreased coagulation factors and a normal result is very unlikely to find any factor deficiency, by direct determination.
\end{abstract}

Key words: Diagnosis, partial thromboplastin time, blood clotting factors. 


\section{Introducción}

El uso del TTPa (tiempo de tromboplastina parcial activado) como prueba de tamización para la detección de pacientes con deficiencias de factores de coagulación ha sido ampliamente usado desde hace ya más de 50 años ${ }^{1}$

Actualmente, es una herramienta importante al momento de valorar pacientes con sospecha de deficiencias de factores, para monitorizar pacientes anticoagulados con heparina y para detección de sujetos con sospecha de anticoagulante lúpico ${ }^{2,3}$.

Sin embargo, la sensibilidad de todos los reactivos usados en esta prueba para detectar deficiencias de factores es variable, por lo cual tradicionalmente se ha recomendado que, ante la alta sospecha clínica de trastorno hemorrágico, se debería solicitar directamente el nivel de actividad del factor de coagulación, considerando la posibilidad de resultados falsos negativos con TTPa 4 .

Esta asunción puede cambiar en la medida en que se han perfeccionado nuevos reactivos que brindan resultados con alta sensibilidad y se ha sugerido que, con el uso de estas pruebas, no es necesaria la realización de estudios de factores de coagulación si el resultado es normal 5 .

La diversidad de métodos ha llevado a múltiples estudios comparativos que han demostrado diferencias en la capacidad para detectar deficiencias según el reactivo empleado ${ }^{6}$. Estos han evidenciado que parece existir una ventaja de Actin FSL comparada con otros reactivos, especialmente por una mayor sensibilidad para la detección de deficiencia de factor VIII (FVIII) y factor IX $(\mathrm{FIX})^{7}$.

De manera contraria, Lawrie y colaboradores ${ }^{8}$ reportaron sensibilidades bajas para Actin FSL con falla para la detección de deficiencias de factor VIII, IX, XI y XII del 5.6, 36.4, 30.4 y $72.2 \%$, respectivamente. No obstante, su metodología es diferente a la actualmente usada en nuestro laboratorio. Recientemente, Bowyer y colaboradores efectuaron un estudio con mezcla de plasmas para obtener diferentes valores de factores y compararlo con cuatro reactivos diferentes, reportando una alta sensibilidad con Actin FS para FVIII y FXI9.

Por lo tanto, está claro que la calidad y tipo de reactivo usado, además de un procedimiento estandarizado, son fundamentales al momento de interpretar los resultados del TTPa cuando se solicita con fines de tamización por sospecha de deficiencias de factores de coagulación.

\section{Métodos}

\section{Objetivos e hipótesis}

\section{Objetivo general}

Determinar la sensibilidad del TTPa con Actin FSL en la detección de pacientes con deficiencia de factores de coagulación comparada con el estándar de referencia, que es la determinación directa del nivel de actividad de factores de coagulación por método coagulométrico de un paso.

\section{Objetivos específicos}

Establecer el mejor punto de corte, de acuerdo con curvas ROC, promedio y dos desviaciones estándar para obtener una sensibilidad cercana al 100\% del TTPa en la detección de un nivel de actividad del factor de coagulación menor del 50\%.

Determinar si existe correlación entre el valor del TTPa en segundos y el nivel de actividad de factores de coagulación.

\section{Tipo de estudio}

Estudio analítico de corte transversal para validación de pruebas diagnósticas.

\section{Selección de la muestra}

Muestra por conveniencia de pacientes del Hospital Militar Central con determinación de TTPa y de niveles de factores de coagulación solicitados por sospecha de trastornos de hemostasia durante el período de junio del 2009 a febrero del 2011. La información se obtuvo de la base de datos y por recopilación de libros de reportes de estudios de coagulación del Laboratorio de Hemostasia y Coagulación del Departamento de Hematología y Oncología Clínica del Hospital Militar Central.

\section{Criterios de inclusión}

Pacientes con determinación en la misma muestra de TTPa y de factores VIII, IX, XI, XII concomitantes, o con TTPa y solo uno de estos factores, siempre y cuando el valor de este último sea inferior al 50\%, realizados en el Laboratorio Especializado de Hemostasia y Coagulación 
del Servicio de Hematología y Oncología del Hospital Militar Central. Se incluyeron todos aquellos pacientes a quienes el médico tratante solicitó dichas pruebas ya sea porque el paciente consultó por síntomas de sangrado anormal o pacientes asintomáticos, pero con prolongación aislada del TTPa durante valoración de otras condiciones médicas o valoraciones prequirúrgicas hechas en un laboratorio diferente.

\section{Criterios de exclusión}

- Pacientes con anticoagulación oral con warfarina, ribaroxaban o dabigatran.

- Pacientes con anticoagulación con heparina no fraccionada y heparina de bajo peso molecular.

- Pacientes con prolongación del INR mayor de 1.3.

\section{Procedimientos realizados}

Se definió disminución del nivel de actividad del factor de coagulación como un valor menor del 50\%, medido por método coagulométrico de un paso. Se consideró deficiencia severa cuando el valor fue inferior al $1 \%$ de actividad; deficiencia moderada entre $1-5 \%$ de actividad; y deficiencia leve cuando el nivel de actividad era mayor de $5 \%$, pero menor de $40 \%$, de acuerdo con las recomendaciones de la Sociedad Internacional de Trombosis y Hemostasia (International Society on Thrombosis and Haemostasis, ISTH $)^{10}$. La determinación de los niveles de actividad de los factores de coagulación se llevó a cabo de manera ciega e independiente a los resultados del TTPa, con el fin de no influenciar los datos de estos resultados.

Las mediciones se realizaron en el equipo CA1500 de Siemens. El TTPa con reactivo Actin FSL se definió como el tiempo que tarda en formar la fibrina después de adicionar una tromboplastina con un activador que, en este caso, es el ácido elágico, incubación de tres minutos y activación del cronómetro cuando se adiciona cloruro de calcio $0.25 \mathrm{M}$. Su valor se midió en segundos.

Dosificación de factores: se efectuó utilizando como base las pruebas de TTP dependiendo del factor que se vaya a titular, se emplea el plasma deficitario, que se mezcla con el plasma del paciente que se va a estudiar, luego se pasa un TTP y los segundos se interpolan en una curva de calibración previamente hecha. El intervalo de referencia de normalidad se estableció entre el 50\% y el 150\%.

\section{Cálculo del tamaño de muestra}

Considerando una sensibilidad esperada del 95\% con una especificidad del $60 \%$ dada por los falsos positivos y pacientes con anticoagulante lúpico, los cuales no analizaremos en este estudio, y asumiendo un nivel de confianza del 95\% con una precisión absoluta del 90\%, el tamaño de muestra esperado fue de 101 pacientes. Al considerar los errores en el laboratorio por pérdida de muestras o datos incompletos, se consideró apropiado un incremento del 10\% para un tamaño de muestra estimado de 111 pacientes.

\section{Plan de análisis}

Se estableció un valor de referencia del TTPa, definido como la media de valores más dos desviaciones estándar, utilizando plasma de 20 sujetos sanos, asintomáticos, sin antecedentes patológicos ni familiares de sangrado y con un valor de FVIII, FIX, FXI, FXII entre el 50-150\%. A partir de este valor, se consideró TTPa prolongado.

Con este valor de referencia de TTPa, se calculó en la población del estudio sensibilidad y LR- (negative likelihood ratio, razón de probabilidades negativo), especificidad y LR+ (positive likelihood ratio, razón de probabilidades positivo).

Adicionalmente, se determinó el mejor punto de sensibilidad por medio de curva ROC. Se realizó análisis por regresión lineal entre el valor de TTPa en segundos y el porcentaje de actividad de los factores de coagulación como variables de razón.

Se utilizó el programa Excel 2010 y Stata versión 11.1 para el análisis estadístico, calculando un intervalo de confianza del 95\% para todos los análisis primarios.

\section{Aspectos éticos}

Estudio no intervencionista sin daño a la salud del paciente, a quien se guardó la confidencialidad de los datos personales; al recolectar información de manera retrospectiva con registros del laboratorio, no se requirió consentimiento informado para la población de estudio. Los 20 sujetos sanos firmaron consentimiento informado. El estudio fue avalado por el Centro de Investigaciones de la Facultad de Medicina de la Universidad Militar Nueva Granada.

\section{Resultados}

Durante el período de junio del 2009 a febrero del 2011, se identificaron 133 pacientes con prueba de TTPa 
por Actin FSL y medición de factores de coagulación de manera concomitante. De estos 133 pacientes incluidos en el análisis, 117 pacientes tenían determinación de FVIII, 85 pacientes determinación de FIX, 74 pacientes determinación de FXI y 68 pacientes determinación de FXII, 54 de ellos tenían determinaciones simultáneas de los cuatro factores. Las características de la población se describen en la tabla 1.

Con la muestra de 20 pacientes sanos, se determinó una media de 29.1 segundos con un límite superior de normalidad de $32 \mathrm{seg}$. dada por dos desviaciones estándar.

Tabla 1. Características demográficas de los sujetos

\begin{tabular}{|l|c|}
\hline \multicolumn{1}{|c|}{ Característica (n= 133) } & $\%$ (n) \\
\hline Género & \\
Mujeres & $56 \%(74)$ \\
Hombres & $44 \%(59)$ \\
\hline Edad & 38 años (18-83) \\
\hline Manifestaciones clínicas & \\
Sospecha de trastornos de la hemostasia por sangrado anormal & $30 \%(40)$ \\
Asintomáticos con TTPa anormal durante valoración prequirúrgica & $40 \%(53)$ \\
Asintomáticos con TTPa anormal por otros motivos & $30 \%(40)$ \\
\hline Pacientes con deficiencia específica de factores (n) & \\
Factor VIII & 32 \\
Deficiencia severa factor VIII & 5 \\
Factor IX & 5 \\
Deficiencia severa factor IX & 1 \\
Factor XI & 13 \\
Factor XII & 14 \\
\hline
\end{tabular}

\section{Rendimiento diagnóstico}

En la población total estudiada, encontramos que, al definir deficiencia de factores como un nivel de actividad $<50 \%$ y un TTPa $>32.1$ segundos, la sensibilidad del TTPa fue del $98.7 \%$ con un LR- de 0.02 (tabla 2) para detectar cualquier factor bajo en toda la población.

Ningún paciente con deficiencia severa de factores de coagulación presentó un valor de TTPa $<32.1 \mathrm{seg}$. El único paciente falso negativo entre los 133 pacientes evaluados fue uno con disminución de factor XII del 44.7\%. Esto llevó a una sensibilidad del 100\% para deficiencia de factores VIII, IX y XI.

La especificidad fue del 51.7 relacionado a que nuestro estudio no evaluó los resultados de anticoagulante lúpico ni la presencia de inhibidores como causa de la prolongación del TTPa. Al realizar la valoración del rendimiento diagnóstico de los factores de coagulación

Tabla 2. Rendimiento diagnóstico con TTPa para deficiencia de cualquier factor VIII, IX, XI o XII (<50\%)

\begin{tabular}{|c|c|c|c|}
\hline Sensibilidad & Especificidad & LR+ & LR- \\
\hline $98 \%$ & $51.7 \%$ & 2.04 & 0.03 \\
IC $95 \%(92.8-99.8 \%)$ & IC 95\%(39.2-64.1) & IC 95\%(1.56-2.67) & IC 95\% (0.00-0.18) \\
\hline
\end{tabular}

Tabla 3. Rendimiento diagnóstico específico de factores de acuerdo con los criterios de la ISTH

\begin{tabular}{|l|c|c|c|c|}
\hline $\begin{array}{c}\text { Factor } \\
(<\mathbf{4 0 \% )}\end{array}$ & Sensibilidad & Especificidad & LR+ & LR- \\
\hline Cualquier factor & $100 \%$ & $42.67 \%$ & 1.7 & $<0.00001$ \\
\hline Factor VIII & $100 \%$ & $30.59 \%$ & 1.44 & $<0.00001$ \\
\hline Factor IX & $100 \%$ & $26.25 \%$ & 1.35 & $<0.00001$ \\
\hline Factor XI & $100 \%$ & $32.79 \%$ & 1.48 & $<0.00001$ \\
\hline Factor XII & $100 \%$ & $33.33 \%$ & 1.50 & $<0.00001$ \\
\hline
\end{tabular}

específicos, siguiendo las definiciones de la ISTH, que determina un umbral de anormalidad como un nivel de actividad del factor de coagulación como menor de $40 \%$, la sensibilidad fue del $100 \%$ en todos los casos (tabla 3).

La evaluación de solo los 54 pacientes con determinaciones simultáneas de los cuatro factores de coagulación evidenció una sensibilidad del 95.5\%, con un falso negativo dado por una disminución leve de factor XII.

La evaluación del rendimiento diagnóstico por curva ROC (receiver operating characteristic, características operativas del receptor) determinó que, con un valor de TTPa de 31.6 seg., no se encontró ningún paciente con deficiencia de factores de coagulación, correspondiente a una sensibilidad del 100\% (figura 1).

El análisis por regresión lineal demostró que no existe correlación entre el porcentaje de actividad de los factores de coagulación y el valor del TTPa en segundos (figura 2).

\section{Discusión}

La prolongación del TTPa en pacientes asintomáticos o encontrar tiempos de coagulación normales en pacientes con manifestaciones hemorrágicas son un motivo frecuente de la consulta hematológica. En el

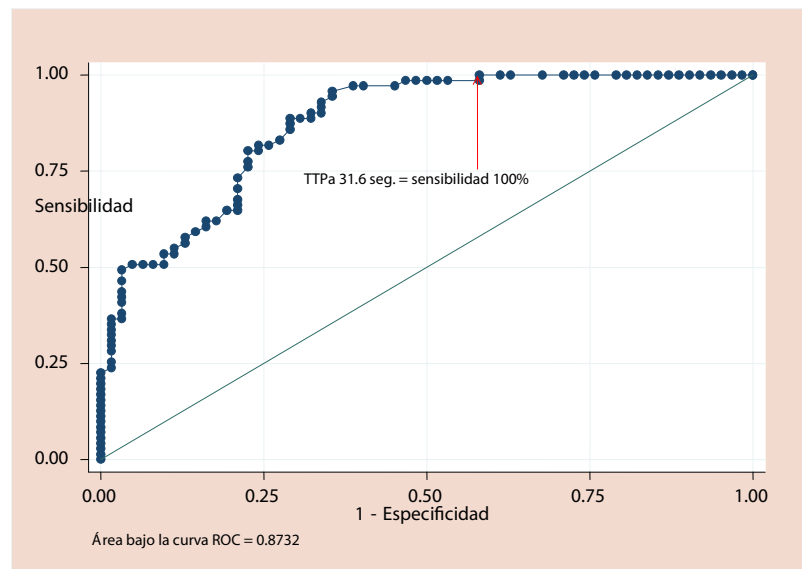

Figura 1. Curva ROC TTPa vs. deficiencia de factores 
Regresión Lineal TTPa con Actividad de Factores de Coagulación

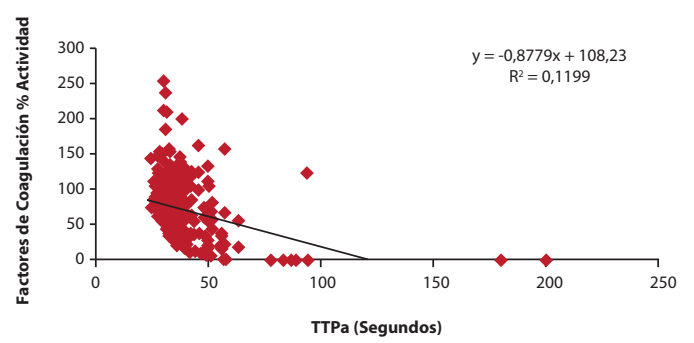

Figura 2. Regresión lineal TTPa vs. factores de coagulación

primer escenario, Chng y colaboradores encontraron que, en pacientes con TTPa prolongado, la principal causa fue la presencia de un anticoagulante lúpico en hasta el $53 \%$ de los casos y solo el $16 \%$ de los casos tenían alguna deficiencia de factores de coagulación como causa de esta prolongación. De manera llamativa, hasta en un $31 \%$ de los casos no se halló una causa de esta anormalidad"11.

En el caso de detección de deficiencia de factores, que fue el objetivo de nuestro estudio, Bowyer y colaboradores reportaron que, de 587 muestras evaluadas, solo 117 fueron anormales y, de estas, el $75 \%$ estaban dadas por deficiencias leves de factor $\mathrm{XI}^{5}$. Estos resultados refuerzan la recomendación de medir los factores de coagulación específicos solo cuando una prueba de TTPa por un método altamente sensible está elevada. Más allá de realizar una evaluación con diferentes pruebas de laboratorio, el enfoque apropiado de los pacientes con verdaderas manifestaciones hemorrágicas anormales depende de un excelente examen clínico, el cual muchas veces es suficiente para orientar con fuerte grado de certeza que las manifestaciones de sangrado podrían deberse a causas diferentes, ya sea de la función y/o cantidad de plaquetas o problemas vasculares más que a deficiencia de proteínas específicas involucradas en el proceso de coagulación $n^{12,13}$.

De igual modo, la evaluación de un TTPa anormalmente prolongado debe seguir una aproximación diagnóstica estructurada basada en consideraciones de la historia clínica, el examen físico y una secuencia de pruebas de laboratorio orientada conforme con los hallazgos del abordaje clínico inicial, más que la realización de múltiples exámenes simultáneos, cuya interpretación posterior puede complicar aún más, de forma innecesaria, el motivo de consulta del paciente ${ }^{14}$.

La principal limitación de nuestro estudio está dada por el carácter retrospectivo en la toma de datos, sin embargo, en este grupo de pacientes, la aplicación del estándar de referencia a todos los pacientes independiente de los resultados de la prueba de tamización (TTPa) refuerza la validez de nuestras conclusiones. Nosotros tampoco consideramos un análisis para determinar la sensibilidad y especificidad en la detección del TTPa acortado por elevación de factor VIII, ni si esto pudiese ser un factor confuso en la interpretación del TTPa medido en segundos, un aspecto que actualmente se está investigando como posible indicador de riesgo trombótico ${ }^{15}$. No obstante, nuestro análisis encontró una pobre correlación entre el tiempo de TTPa en segundos y el valor de los factores de coagulación $\left(r^{2}=0.11\right)$, un resultado diferente al de Boekel y colaboradores, que demostraron una importante asociación entre el acortamiento del TTPa y la elevación del FVIIII'. Este objetivo requeriría una metodología de diseño y analítica diferente para corroborar dichos hallazgos. En este mismo sentido, no encontrar una relación lineal entre el valor del TTPa en segundos y el porcentaje de actividad del factor de coagulación desvirtúa completamente el uso del TTPa como método de monitoreo indirecto para pacientes con deficiencias confirmadas, por ejemplo, con hemofilia, en quienes tomar conductas terapéuticas basadas en el TTPa conllevaría a un enorme riesgo de error. Otro punto por considerar es el cambio del TTPa según la edad de la población analizada, un factor que no se evaluó en nuestro estudio, debido a que tal prolongación parece estar relacionada de manera directa con diferentes causas de esta anormalidad, más que con un factor biológico primario entre edad y valor del TTPa ${ }^{17}$.

La pobre especificidad de la prueba del $51 \%$ está explicada por la posible presencia de pacientes con inhibidores específicos o anticoagulante lúpico. Esta medición, sin embargo, no se realizó en todos los pacientes $y$, por lo tanto, no la reportamos, debido a que el objetivo de nuestro estudio fue determinar la sensibilidad de la prueba como método de tamización para detectar deficiencia de factores de coagulación y no para evidenciar las causas de prolongación de TTPa. 
Nuestros resultados demuestran claramente que, bajo una técnica estandarizada, el uso de TTPa con el reactivo de Actin FSL tiene una sensibilidad cercana al 100\% en la detección de deficiencias severas y de más del $98 \%$ en deficiencias leves. Esta sensibilidad menor del $2 \%$ de falsos negativos se observó específicamente para deficiencias leves de factor XII, lo cual, desde nuestra percepción, tiene poca relevancia clínica, debido a que este tipo de disminuciones no ubican al paciente en riesgo incrementado de eventos hemorrágicos.

Desde un punto de vista aplicativo, considerando en el peor escenario de rendimiento diagnóstico un LR- de 0.03 , en pacientes con una probabilidad pretest del $5 \%$ de tener un trastorno de coagulación por deficiencia de factores (hemofilia congénita o adquirida), la probabilidad postest sería menor del $1 \%$ si se obtiene un valor de TTPa normal; en pacientes con probabilidad pretest del $50 \%$, un TTPa normal cambiaría la probabilidad postest a solo un 2\%; y únicamente en el caso de una muy alta probabilidad pretest del $90 \%$, encontrar un TTPa normal con Actin FSL resultaría en una probabilidad postest del 15\% (un escenario clínico extremadamente improbable debido a que la sensibilidad para deficiencias severas fue del 100\%).

\section{Conclusiones}

En pacientes con TTPa medido con Actin FSL bajo una técnica estandarizada, un resultado menor de 32 segundos hace innecesaria la titulación de ensayos específicos de factores de coagulación, y, por lo tanto, no se recomienda de manera rutinaria. Si bien la indicación puede sonar controversial, estos pacientes muy seguramente tendrían una causa diferente de sus manifestaciones de hemostasia anormal, y, por ende, su abordaje diagnóstico debería considerar otras etiologías y métodos diagnósticos alternativos.

El valor del TTPa no se correlaciona con el porcentaje de actividad de los factores de coagulación, por lo cual el TTPa si bien es útil como prueba de tamización, no lo es para monitorizar pacientes con deficiencia de factores.

\section{Abreviaciones}

TTPa (tiempo de tromboplastina parcial activado), FVIII (factor VIII), FIX (factor IX), FXI (factor XI), FXII (factor XII), LR- (negative likelihood ratio, razón de probabilidades negativo), LR+ (positive likelihood ratio, razón de probabilidades positivo), curva ROC (receiver operating characteristic, características operativas del receptor).

\section{Referencias}

1. Proctor RR, Rapaport SI. The partial thromboplastin time with kaolin. A simple screening test for first stage plasma clotting factor deficiencies. Am J Clin Pathol. 1961;36:212-9.

2. Chee $Y L$, Crawford JC, Watson HG, Greaves M. Guidelines on the assessment of bleeding risk prior to surgery or invasive procedures. British Committee for Standards in Haematology. Br J Haematol. 2008;140(5):496-504.

3. Pengo V, Tripodi A, Reber G, Rand JH, Ortel TL, Galli M, et al. Update of the guidelines for lupus anticoagulant detection. Subcommittee on Lupus Anticoagulant/Antiphospholipid Antibody of the Scientific and Standardisation Committee of the International Society on Thrombosis and Haemostasis. J Thromb Haemost. 2009;7(10):1737-40.

4. Kitchen SM, Echenagucia M. Diagnosis of hemophilia and other bleeding disorders. Montréal, Québec: the World Federation of Hemophilia (WFH); 2010

5. Bowyer A, Smith J, Woolley AM, Kitchen S, Hampton KK, Maclean RM, et al. The investigation of a prolonged APTT with specific clotting factor assays is unnecessary if an APTT with Actin FS is normal. Int J Lab Hematol. 2011;33(2):212-8.

6. Brandt JT, Arkin CF, Bovill EG, Rock WA, Triplett DA. Evaluation of APTT reagent sensitivity to factor IX and factor IX assay performance. Results from the College of American Pathologists Survey Program. Arch Pathol Lab Med. 1990;114(2):135-41.
7. Shetty S, Ghosh K, Mohanty D. Comparison of four commercially available activated partial thromboplastin time reagents using a semi-automated coagulometer. Blood Coagul Fibrinolysis. 2003;14(5):493-7.

8. Lawrie AS, Kitchen S, Purdy G, Mackie IJ, Preston FE, Machin SJ. Assessment of Actin FS and Actin FSL sensitivity to specific clotting factor deficiencies. Clin Lab Haematol. 1998;20(3):179-86.

9. Bowyer $A$, Kitchen $S$, Makris $M$. The responsiveness of different APTT reagents to mild factor VIII, IX and XI deficiencies. Int J Lab Hematol. 2011;33(2):154-8.

10. White GC 2nd, Rosendaal F, Aledort LM, Lusher JM, Rothschild $C$, Ingerslev J. Definitions in hemophilia. Recommendation of the scientific subcommittee on factor VIII and factor IX of the scientific and standardization committee of the International Society on Thrombosis and Haemostasis. Thromb Haemost. 2001;85(3):560

11. Chng WJ, Sum C, Kuperan P. Causes of isolated prolonged activated partial thromboplastin time in an acute care general hospital. Singapore Med J. 2005;46(9):450-6.

12. Girolami A, Luzzatto G, Varvarikis $C$, Pellati $D$, Sartori R, Girolami B. Main clinical manifestations of a bleeding diathesis: an often disregarded aspect of medical and surgical history taking. Haemophilia. 2005;11(3):193-202. 
13. Favaloro EJ. Investigating people with mucocutaneous bleeding suggestive of primary hemostatic defects: a low likelihood of a definitive diagnosis? Haematologica. 2007;92(3):292-6.

14. Kamal AH, Tefferi A, Pruthi RK. How to interpret and pursue an abnormal prothrombin time, activated partial thromboplastin time, and bleeding time in adults. Mayo Clin Proc. 2007;82(7):864-73.

15. Ng VL. Prothrombin time and partial thromboplastin time assay considerations. Clin Lab Med. 2009;29(2):253-63.
16. Ten Boekel E, Bock M, Vrielink GJ, Liem R, Hendriks H, de Kieviet $\mathrm{W}$. Detection of shortened activated partial thromboplastin times: an evaluation of different commercial reagents. Thromb Res. 2007;121(3):361-7.

17. Li J, Lai X, Yan C, Xu A, Nie L, Zhou Y, et al. Age-associated developmental changes in the activated partial thromboplastin time (APTT) and causes of prolonged APTT values in healthy Chinese children. Clin Chem Lab Med. 2009;47(12):1531-7. 